\title{
Análise da capacidade funcional pelo Six Minute Walk Test (6MWT) em doentes renais crônicos
}

\author{
Functional capacity analysis using the Six Minute Walk \\ Test (6MWT) in chronic renal patients
}

\section{Fabiana Santos Franco', Maria Eduarda Freitas Caiado Fleury², Marcos Mello Moreira3 Patrícia Leão da Silva Agostinho4, Giulliano Gardenghi ${ }^{5}$}

\author{
'Universidade Federal de Jataí. Jataí, Goiás, Brasil. ORCID: 0000-0002-1784-8580. dra_fabianafranco@hotmail.com \\ Universidade Federal de Jataí. Jataí, Goiás, Brasil. ORCID: 0000-0001-7252-9833. eduardafleury@outlook.com \\ ${ }^{3}$ Universidade Estadual de Campinas. Campinas, São Paulo, Brasil. ORCID: 0000-0002-2148-5479. marcosmm@fcm.unicamp.br \\ ${ }^{4}$ Universidade Federal de Jataí. Jataí, Goiás, Brasil. ORCID: 0000-0003-2694-0247. p.leao@hotmail.com
}

${ }^{5}$ Autor para correspondência. Centro de Estudos Avançados e Formação Integrada (Goiânia), Hospital Encore (Aparecida de Goiânia). Goiás, Brasil. ORCID: 0000-0002-8763-561X. coordenacao.cientifica@ceafi.com.br

\begin{abstract}
RESUMO | INTRODUÇÃO: A doença renal crônica (DRC) é caracterizada por alterações na função ou estrutura renal durante 3 meses ou mais, levando a perda funcional dos rins e a diminuição da capacidade funcional. OBJETIVO: Avaliar a capacidade física de pacientes doentes renais crônicos no estagiamento 5, através do six minute walk test (6MWT), e comparar a distância percorrida e prevista de acordo o gênero. MÉTODOS: Trata-se de um estudo transversal, de caráter analítico descritivo. Foram incluídos indivíduos com DRC no estagiamento 5, de 18 a 59 anos, de ambos os gêneros e excluídos pacientes com deformidades em membros inferiores, tabagistas, etilistas e com doença pulmonar obstrutiva crônica. Os participantes foram submetidos ao six minute walk test. Foram realizados os cálculos de média e desvio padrão, os testes de Pearson e T-student, respeitando o valor de significância $<0,05$ para análise estatística através do software SPSS 20.0 para Windows. RESULTADOS: Dos 139 pacientes em hemodiálise, 30 foram elegíveis, sendo que destes 12 (doze) eram do gênero feminino e 18 (dezoito) do gênero masculino, com idade média em anos de $42,33 \pm 15,58$ e 39,27士12,22. A distância prevista em metros para o gênero feminino foi de $763,10 \pm 63,62$ e gênero masculino $840,82 \pm 52,42$. A média de distância percorrida pelo gênero feminino foi de $325,33 \pm 78,31$ ( $r=0,1, p=0,00$ ), do gênero masculino $389,67 \pm 95,80$ metros ( $r=0,98, p=0,00$ ). CONCLUSÃO: Indivíduos renais crônicos no estagiamento 5, apresentam desempenho inferior ao previsto de acordo com a distância prevista e percorrida através 6MWT independente do gênero, indicando uma menor capacidade funcional desta população.
\end{abstract}

PALAVRAS-CHAVE: Doença renal crônica. Atividades cotidianas. Teste de caminhada.
ABSTRACT | INTRODUCTION: Chronic kidney disease (CKD) is characterized by changes in kidney function or structure for 3 months or more, leading to functional loss of the kidneys and decreased functional capacity. OBJECTIVE: To evaluate the physical capacity of patients with chronic kidney disease during stage 5 , using the six minute walk test (6MWT), and to compare the distance covered and predicted according to gender. METHODS: This is a cross-sectional, descriptive analytical study. Individuals with CKD were included in stage 5, aged 18 to 59 years, of both genders and patients with lower limb deformities, smokers, alcoholics and chronic obstructive pulmonary disease were excluded. Participants underwent the six minute walk test. Mean and standard deviation calculations were performed, as well as the Pearson and T-student tests, respecting the significance value $<0.05$ for statistical analysis using the SPSS 20.0 software for Windows. RESULTS: Of the 139 patients on hemodialysis, 30 were eligible, of which 12 (twelve) were female and 18 (eighteen) male, with a mean age in years old of 42.33 \pm 15.58 and $39.27 \pm 12,22$. The predicted distance in meters for females $763.10 \pm 63.62$ and males $840.82 \pm 52.42$. The average distance covered by the female gender was $325.33 \pm 78.31(r=0.1, p=0.00)$, male $389.67 \pm$ 95.80 meters $(r=0.98, p=0.00)$. CONCLUSION: Chronic renal individuals undergoing staging 5 , perform less than expected according to the predicted distance and traveled through 6MWT regardless of gender, indicating a lower functional capacity of this population.

KEYWORDS: Chronic kidney disease. Daily activities. Walk test. 


\section{Introdução}

De acordo com a National Kidney Fundation, a doença renal crônica (DRC) é caracterizada por alterações na função ou estrutura renal durante 3 meses ou mais, de caráter assintomático e progressivo, levando a perda funcional dos rins, sendo necessário realizar terapia substitutiva, por meio de diálise peritoneal ou hemodiálise, estabelecendo um equilíbrio metabólico e nutricional ${ }^{1,2}$.

Estima-se que no Brasil, cerca de mais de dois miIhões de indivíduos possuam alguma disfunção renal, e, cem mil deles realizem terapia de substituição renal, a detecção precoce é fundamental, pois atua na evolução natural da doença diminuindo as complicações e necessidades de realizar terapia substitutiva $^{3}$. De acordo com a Sociedade Brasileira de Nefrologia, 91,4\% das pessoas que necessitam de terapia de substituição renal (TSR) são submetidos à hemodiálise, que apesar de proporcionar melhoras ao paciente em estado crítico, pode acarretar outras complicações como instabilidade hemodinâmica, cateteres venosos, sangramento devido à administração de heparina, além de restrições e limitações das atividades de vida diária do paciente no momento em que o tratamento é iniciado ${ }^{4,5}$.

A doença renal apresenta elevadas taxas de sobrevida, entretanto, é considerada uma doença altamente intrusiva devido às complicações e restrições que podem proporcionar, juntamente com a incerteza do seu prognóstico, esses fatores são somados e potencializados devido a instabilidade familiar, pessoal e laboral no qual o doente se encontra ${ }^{3,6}$. A DRC apresenta 5 estágios os quais são classificados de acordo com a fase da doença e sua causa, além das anormalidades no sedimento urinário, alteração em exame de imagem renal e determinação da taxa filtração glomerular (TFG) ${ }^{1,7}$. A última fase, conhecida como crônica é representada com a incompatibilidade dos rins com a vida, perdendo totalmente a sua função e o controle com o meio interno, sendo necessário realizar terapia de substituição renal ou transplante, compreendendo a uma TFG inferior à $15 \mathrm{ml} / \mathrm{min} / 1.73 \mathrm{m2}^{7}$.
A grande parte dos pacientes com DRC estão entre os estágios 3 e 5, onde o declínio da TFG está ligado a diminuição do desempenho físico, acarretando a apresentarem modificações da função física devido à doença, com descondicionamento e baixa tolerância às atividades e redução da capacidade funcional. Todavia, depois de iniciada a hemodiálise é comum manifestar, fadiga, câimbras e prostrações ${ }^{3,8}$, além de casos de sarcopenia ${ }^{5,9,10}$, gerando um impacto no sistema musculoesquelético, levando a hipotrofia, atrofia muscular e até mesmo fraqueza generalizada que afetam a funcionalidade, a independência e o bem estar geral dos indivíduos ${ }^{11}$.

A avaliação de alterações fisiológicas pode ser realizada a partir do six minute walk test (6MWT), que é uma ferramenta muito utilizada para avaliar a capacidade funcional de pessoas ativas, sedentárias e portadores de doenças crônicas. Este teste avalia a intensidade suficiente para atingir a capacidade aeróbica máxima a partir da distância percorrida, onde o consumo de oxigênio (VO2) corresponde a um exercício submáximo, no qual VO2 atinge um platô, mas não chega a um valor máximo, fornecendo uma resposta integrada de todos os sistemas envolvidos para executar um exercício ${ }^{12,14}$.

Entretanto, atualmente na literatura há escassez de dados que corroborem ou não a correlação entre os valores inferiores apresentados no 6MWT em relação ao esperado no teste e as disfunções causadas devido a DRC. Assim, o objetivo deste trabalho foi avaliar o desempenho de pacientes doentes renais crônicos no estagiamento 5 , através do 6MWT e correlacionar com sua capacidade física, além de comparar a distância percorrida com a distância prevista de acordo o gênero.

\section{Materiais e métodos}

Trata-se de uma pesquisa do tipo transversal, observacional, com abordagem analítica descritiva, aprovada pelo comitê de ética e pesquisa (CAAE 69175717.8.0000.5083), a qual foi desenvolvida em um Centro de Uro-nefrologia, onde os pacientes 
responderam a um questionário sociodemográfico e foram submetidos ao 6MWT no momento pré-diálise para avaliação da capacidade funcional no período de setembro a dezembro de 2018.

Foram inclusos os pacientes com DRC no estagiamento 5, com 18 a 59 anos de idade, de ambos os gêneros, hemodinamicamente estáveis e que apresentem condições clínicas estáveis que permitisse a realização do teste físico. Foram excluídos da pesquisa, pacientes que apresentam deformidades em membros inferiores, pacientes tabagistas, etilistas, com doença pulmonar obstrutiva crônica não apresentassem aumento de peso entre diálise >2,5kg.

O teste foi realizado em um corredor de comprimento mínimo de 30 metros de extensão, plano e livre do trânsito de pessoas, com familiarização e reconhecimento do percurso antes do seu início, foram realizadas demarcações de 3 em 3 metros e também foi disponibilizada uma cadeira caso o paciente necessite de apoio antes de terminar todo o trajeto ${ }^{14,15}$.

Ao início e final do teste foi avaliado as variáveis de frequência cardíaca ( $F C)$, frequência respiratória (FR), distância percorrida (DP), sensação subjetiva de dispneia aplicado a escala de Borg modificada ${ }^{15}$. A distância caminhada prevista (\%) no 6MWT foi calculada de acordo com a equação para a população brasileira desenvolvida por Britto ${ }^{14}$.

Após a coleta dos dados, os testes estatísticos foram realizados no software SPSS versão 20.0 para o Windows. Os dados são apresentados com média (+) ou desvio (-) padrão. Para a análise da normalidade dos dados foi aplicado o teste de Shapiro-Wilk e posteriormente foi utilizado nas análises de comparação o teste T Student. Para as análises de correlação foi utilizado o teste de Pearson. Foi considerado como valor estatisticamente significante $p<0.05$.

\section{Resultados}

Dos 139 pacientes em hemodiálise do Centro de hemodiálise de Jataí-GO, 30 foram elegíveis (Figura 1), com idade de 18 a 59 anos, sendo que destes 12 (doze) são do gênero feminino e 18 (dezoito) do gênero masculino (Tabela 1).

Figura 1. Fluxograma de recrutamento da amostra

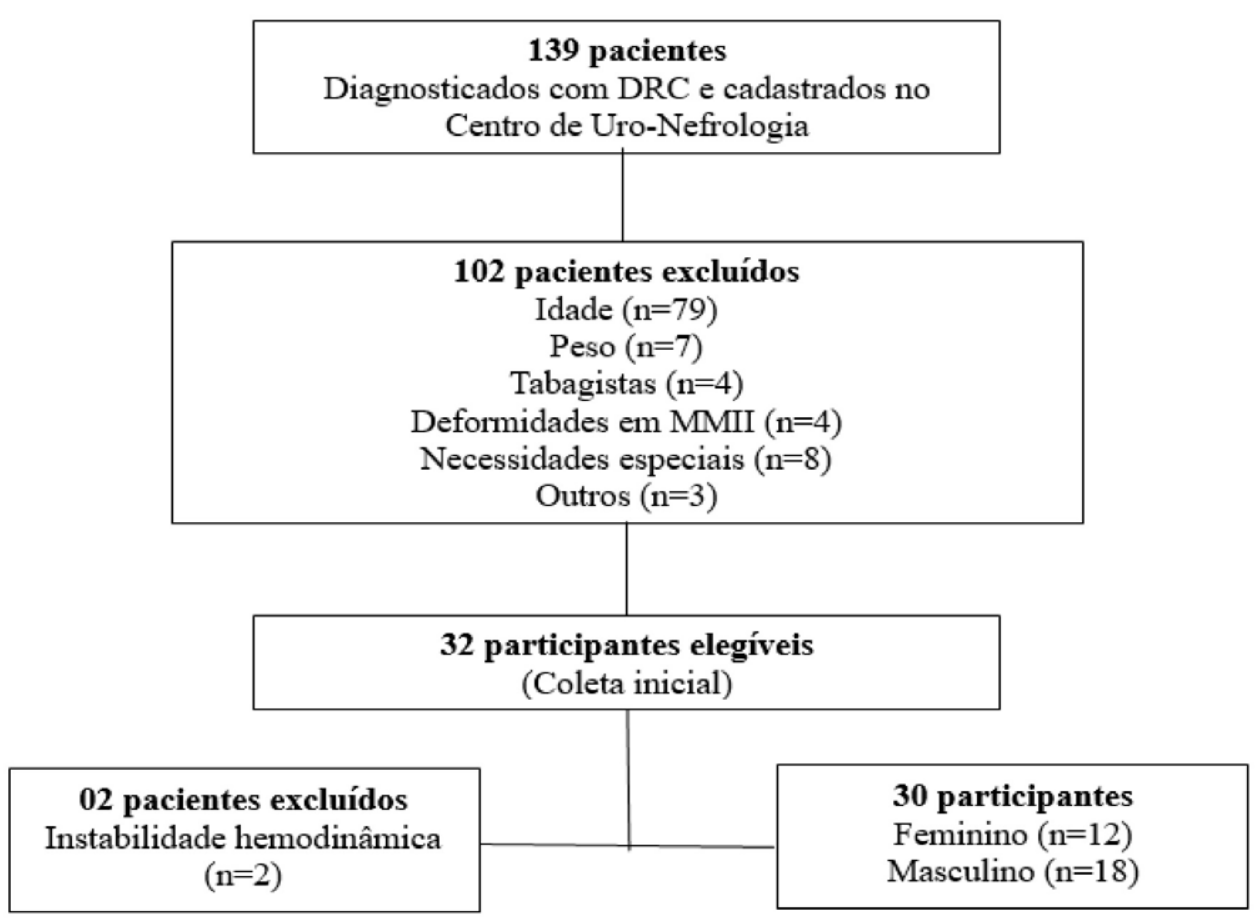


Tabela 1. Caracterização da amostra referente a uma população de 30 indivíduos com DRC, distribuída de acordo com o gênero, idade, altura, tempo de diálise e peso no ano de 2018

\begin{tabular}{|c|c|c|c|c|}
\hline VARIÁVEIS & $\begin{array}{l}\text { Gênero Feminino } \\
\qquad N=12\end{array}$ & $\begin{array}{c}P \\
<0,05\end{array}$ & $\begin{array}{l}\text { Gênero masculino } \\
\qquad N=18\end{array}$ & $\begin{array}{c}P \\
<0,05\end{array}$ \\
\hline Idade & $42,33 \pm 15,58$ & 0,00 & $39,27 \pm 12,22$ & 0,00 \\
\hline Altura em cm & $153,41 \pm 9,95$ & 0,00 & $170,94 \pm 7,36$ & 0,00 \\
\hline Tempo de hemodiálise (meses) & $44,09 \pm 37,64$ & 0,03 & $38,77 \pm 27,00$ & 0,00 \\
\hline Peso em kg & $56,58 \pm 11,45$ & 0,00 & $71,11 \pm 13,13$ & 0,00 \\
\hline Peso seco em kg & $55,18 \pm 10,85$ & 0,00 & $69,52 \pm 13,06$ & 0,00 \\
\hline IMC peso seco & $23,54 \pm 4,83$ & 0,00 & $23,77 \pm 4,07$ & 0,00 \\
\hline
\end{tabular}

Legenda: kg - quilograma, m - metros. Test t-student.

Quando realizada a distribuição referente aos gêneros e realizado o pareamento variáveis descritas na tabela 2 e 0 6MWT, observa-se o resultado em metros para a distância prevista para o gênero feminino 763,10 $\pm 63,62$ e gênero masculino $840,82 \pm 52,42$, e para a qual a média de distância percorrida pelo gênero feminino foi de $325,33 \pm 78,31$ $(r=0,1, p=0,00)$, e a média do gênero masculino 389,67 $\pm 95,80(r=0,98, p=0,00)$. Nota-se que a distância percorrida apresenta valores inferiores a distância predita, independentemente do gênero, como mostra a tabela 2.

Quando analisa a distribuição da amostra de acordo com o gênero e pareadas ao 6MWT (tabela 2), nota-se que os resultados da distância percorrida são inferiores a distância prevista para as variáveis estudadas. Ainda foi possível observar uma correlação negativa moderada entre a distância percorrida em metros e a idade de ambos gêneros. Quando realizado o pareamento do IMC e os valores do mesmo teste, observou-se uma correlação negativa moderada para o gênero feminino e fraca para o masculino.

Observa-se assim, que independente do gênero, indivíduos dialíticos apresentam uma distância percorrida inferior à distância predita quando submetidos ao 6MWT e avaliados de acordo com equação brasileira, indicando assim, uma menor capacidade funcional destes indivíduos.

Tabela 2. Amostras pareadas de acordo com o gênero e 6MWT de 30 indivíduos com DRC no ano de 2018

\begin{tabular}{|c|c|c|c|c|c|c|}
\hline VARIÁVEIS & $\begin{array}{c}\text { Gênero Feminino } \\
\qquad N=12\end{array}$ & $\mathrm{R}$ & $\begin{array}{c}P \\
<0,05\end{array}$ & $\begin{array}{c}\text { Gênero masculino } \\
\qquad N=18\end{array}$ & $\mathrm{R}$ & $\begin{array}{c}P \\
<0,05\end{array}$ \\
\hline Distância prevista & $763,10 \pm 63,62$ & & 0,00 & $840,82 \pm 52,42$ & & 0,00 \\
\hline Distância percorrida (m) & $325,33 \pm 78,31$ & 0,16 & 0,00 & $389,67 \pm 95,80$ & 0,98 & 0,00 \\
\hline Altura em cm & $171,91 \pm 78,77$ & 0,17 & 0,00 & $218,72 \pm 94,23$ & 0,25 & 0,00 \\
\hline Peso seco (kg) & $270,15 \pm 83,17$ & $-0,39$ & 0,00 & $320,14 \pm 99,50$ & $-0,20$ & 0,00 \\
\hline Peso (kg) & $268,75 \pm 83,44$ & $-0,39$ & 0,00 & $318,56 \pm 99,32$ & $-0,22$ & 0,00 \\
\hline Idade & $283,00 \pm 86,80$ & $-0,47$ & 0,00 & $350,39 \pm 101,32$ & $-0,40$ & 0,00 \\
\hline IMC & $301,10 \pm 80,50$ & $-0,45$ & 0,00 & $365,89 \pm 97,35$ & $-0,36$ & 0,00 \\
\hline $\begin{array}{l}\text { Tempo de diálise } \\
\text { (meses) }\end{array}$ & $283,54 \pm 93,34$ & $-0,10$ & 0,00 & $350,89 \pm 97,74$ & 0,06 & 0,00 \\
\hline
\end{tabular}

Legenda: kg - quilograma, m - metros. Teste de Pearson e Test t-student. 


\section{Discussão}

Apesar da amostra deste estudo ser reduzida, os resultados demonstram que o 6MWT é hábil para avaliar a capacidade funcional de indivíduos portadores de DRC.

Estudos anteriores têm demonstrado que a capacidade funcional, que é definida pelas condições necessárias para o indivíduo viver de maneira autônoma e desempenhar de forma satisfatória as atividades de vida diária, sofre alterações associadas ao estado de uremia de pacientes dialíticos, que incluem fraqueza muscular e distúrbios da mecânica respiratória16,17.

Quando comparada a distância percorrida com a distância prevista, observaram-se valores inferiores a esta última variável. Foi possível verificar ainda que independente do gênero, os valores percorridos são menores, embora a distância percorrida pelo gênero masculino tenha sido maior quando comparada a feminina. Isso pode em parte ser justificado pelo dano tecidual causado pelo estresse oxidativo ${ }^{18}$.

Em outros estudos, também observaram a redução das distâncias percorridas por pacientes dialíticos, concordando com os nossos achados. Sugerindo assim que a própria diálise é um fator contribuinte para a redução da capacidade funcional física em pacientes dialíticos, devido à maioria destes indivíduos apresentares diminuição da variabilidade da frequência cardíaca (VFC), bem como a presença de comorbidades advindas da doença e do tratamento, como a diminuição de força muscular, câimbras e fadiga, que são limitantes para a prática de atividade física ${ }^{4,8}$.

Foram analisados os dados do IMC em relação à distância percorrida no 6MWT, os quais indicam que quanto menores este índice, maior será a distância percorrida no teste, uma vez que o mesmo corresponde a massa livre de gordura no indivíduo, influenciando diretamente na capacidade físi$\mathrm{ca}^{19,20}$, sugerindo a existência de prejuízos maiores na distância percorrida com elevados níveis de IMC. No entanto, mesmo a amostra tendo apresentado um IMC menor, a distância percorrida ainda foi inferior à distância predita, justificada pelo fato de dialíticos apresentarem redução do condicionamento físico e da capacidade funcional ${ }^{20}$.
Este estudo demonstrou também, que quanto menor a idade, maior será a distância percorrida, que é atribuído devido ao declínio funcional existente conforme ocorre o avanço da idade, promovendo a diminuição da força muscular, decorrentes do processo natural de envelhecimento ${ }^{21}$. Esta apresenta fatores levam à alteração nas fibras musculares tipo II, redução do leito vascular e capilar, diminuição do fluxo sanguíneo local, contribuindo assim para alterações musculares ${ }^{22}$.

Desta forma, as mudanças metabólicas em pacientes submetidos à hemodiálise geram, portando, prejuízo da capacidade funcional23, visto que a DRC afeta de forma seletiva fibras nervosas de grande calibre ${ }^{17}$. A retenção de toxinas urêmicas vai além da sobrecarga muscular, evidenciando disfunção musculoesquelética, perda da capilaridade, perda de proteínas contráteis e diminuição da massa muscular ${ }^{24,25}$.

Esse estudo possui limitações que devem ser consideradas. Em nossa investigação podemos citar o pequeno tamanho amostral e a inexistência de um grupo controle sem a DRC.

\section{Conclusão}

O 6MWT é um método de avaliação significativo, de fácil aplicabilidade, baixo custo, podendo ser utilizado para avaliação da capacidade funcional de doentes renais crônicos. Com este estudo pode-se observar que indivíduos renais crônicos no estagiamento 5 apresentam desempenho inferior ao previsto considerando a distância percorrida no 6MWT, independente do gênero, o que indica uma menor capacidade funcional desta população.

\section{Agradecimentos}

Agradecemos ao Centro de Uro-nefrologia e Clínica de Hemodiálise, a Universidade Federal de Goiás-Regional Jataí, aos profissionais e participantes que colaboraram na realização deste estudo. 


\section{Contribuições dos autores}

Franco FS participou da concepção, delineamento, busca e análise estatística dos dados da pesquisa, interpretação dos resultados e redação do artigo científico. Fleury MEFC participou da interpretação dos dados, redação do artigo. Moreira MM participou da redação do artigo científico. Agostinho PLS, participou da concepção, delineamento, busca. Gardenghi G participou da redação do artigo científico.

\section{Conflitos de interesses}

Nenhum conflito financeiro, legal ou político envolvendo terceiros (governo, empresas e fundações privadas, etc.) foi declarado para nenhum aspecto do trabalho submetido (incluindo, mas não se limitando a subvenções e financiamentos, participação em conselho consultivo, desenho de estudo, preparação de manuscrito, análise estatística, etc.).

\section{Referências}

1. Effects of daily inspiratory muscle training on respiratory muscle strength and chest wall regional volumes in hemodialysis patients: a randomised clinical trial. Disabil Rehabil. 2019;41(26):3173-3180. doi: 10.1080/09638288.2018.1485181

2. Belangero VMS, Prates LC, Watanabe A, Schvartsman BSG, Nussenzveig $P, C r u z$ NA et al. Prospective cohort analyzing risk factors for chronic kidney disease progression in children. J Pediatr. 2018;94(5):525-31. doi: 10.1016/j.jped.2017.07.015

3. Vanelli CP, Paula RB, Costa MB, Bastos MG, Miranda LSP, Colugnati FAB. Chronic kidney disease: Susceptibility in a representative population-based sample. Rev Saude Publica. 2018;52:1-4. doi: $10.11606 /$ S1518-8787.2018052017410

4. Oliveira ACF, Vieira DSR, Bündchen DC. Nível de atividade física e capacidade funcional de pacientes com doença renal crônica pré-dialítica e em hemodiálise. Fisioter e Pesqui. 2018;25(3):323-9. doi: 10.1590/1809-2950/18003625032018

5. Teles F, Santos RO, Lima HMAM, Campos RP, Teixeira EC, Alves ACA et al. The impact of dialysis on critically ill elderly patients with acute kidney injury: an analysis by propensity score matching. J Bras Nefrol. 2019;41(1):14-21. doi: 10.1590/2175-8239jbn-2018-0058

6. Ferreira PL, Anes EJ. Medição da qualidade de vida de insuficientes renais crónicos: criação da versão portuguesa do KDQOL-SF. Rev Port Saúde Pública. 2010;28(1):31-39.
7. Montenegro MR, Walter RM, Morimoto J, Paternez ACAC. Correlação dos métodos de avaliação nutricional de pacientees submetidos a hemodiálise. Rev Saúde e Pesqui. 2015;8(2):267-75. doi: $10.17765 / 1983-1870.2015$ v8n2p267-275

8. Reboredo MM, Pinheiro BV, Neder JA, Ávila MPW, Ribeiro MLBA, Mendonça AF et al. Effects of aerobic training during hemodialysis on heart rate variability and left ventricular function in end-stage renal disease patients. J Bras Nefrol. 2010;32(4):372-379. doi: 10.1590/S0101-28002010000400006

9. Hellberg M, Höglund P, Svensson P, Clyne N. Randomized Controlled Trial of Exercise in CKD-The RENEXC Study. Kidney Int Reports. 2019;4(7):963-76. doi: 10.1016/j.ekir.2019.04.001

10. Dino BD, Campos R. Insuficiência renal crônica e suas implicações para os renal chronic disease and its implication to the metabolic systems. Rev Uniandrade. 2018;18(3):149-56. doi: 10.18024/1519-5694/revuniandrade.v18n3p149-156

11. Fassbinder TRC, Winkelmann ER, Schneider J, Wendland J, Oliveira OB. Functional Capacity and Quality of Life in Patients with Chronic Kidney Disease In Pre-Dialytic Treatment and on Hemodialysis - A Cross sectional study. J Bras Nefrol. 2015;37(1):47-54. doi: 10.5935/0101-2800.20150008

12. Mancuzo EV, Soares MR, Pereira CAC. Six-minute walk distance and survival time in patients with idiopathic pulmonary fibrosis in Brazil. J Bras Pneumol. 2018;44(4):267-72. doi: 10.1590/s180637562018000000049

13. Vásquez-Gómez J, Castillo-Retamal M, Carvalho RS, FaundezCasanova C, Portes Junior MDP. Prueba De Caminata De Seis Minutos ¿Es Posible Predecir El Consumo De Oxígeno En Personas Con Patologías? Una Revisión Bibliográfica. MHSalud. 2017;16(1). doi: $10.15359 /$ mhs.16-1.1

14. Britto RR, Probst VS, Dornelas AFA, Samora GAR, Hernandes NA, Marinho PEM et al. Reference equations for the six-minute walk distance based on a Brazilian multicenter study. Braz J Phys Ther. 2013;17(6):556-63. doi: 10.1590/\$1413-35552012005000122

15. Amelia MAM, Barros Filho RJS, Almeida MM, Almeida IM. Test of six-minute walk : a situational analy' sis of your practice. 2015;1(1):39-46.

16. Morales-Blanhir JE, Vidal CDP, Romero M de JR, Castro MMG, Villegas AL, Zamboni M. Six-minute walk test: a valuable tool for assessing pulmonary impairment. J Bras Pneumol. 2011;37(1):110-117.

17. Olaizola I, Caorsi H, Fajardo L, Ferreiro A, Campistrus N, Dolinsky $D$ et al. Effectiveness and safety of a 6-month treatment with paricalcitol in patients on hemodialysis with secondary hyperparathyroidism. J Bras Nefrol. 2016;38(3):302-12. doi: 10.5935/0101-2800.20160047 
18. Jaqueto M, Delfino VDA, Bortolasci CC, Barbosa DS, Morimoto $\mathrm{HK}$, Frange RFN et al. Are PTH levels related to oxidative stress and inflammation in chronic kidney disease patients on hemodialysis? J Bras Nefrol. 2016;38(3):288-95. doi: 10.5935/0101$\underline{2800.20160045}$

19. Jacques PS, Gazzana MB, Palombini DV, Barreto SSM, Dalcin PTR. Six-minute walk distance is not related to quality of life in patients with non-cystic fibrosis bronchiectasis. J Bras Pneumol. 2012;38(3):346-55.

20. Teixeira CG, Duarte MC, Prado CM, Albuquerque EC, Andrade LB. Impact of chronic kidney disease on quality of life, lung function, and functional capacity. J Pediatr. 2014;90(6):580-6. doi: 10.1016/j.jped.2014.03.002

21. Sperandio EF, Guerra RLF, Romiti M, Gagliardi ART, Arantes RL, Dourado VZ. Reference values for the 6-min walk test in healthy middle-aged and older adults: from the total distance traveled to physiological responses. Fisioter em Mov. 2019;32:e003231. doi: 10.1590/1980-5918.032.ao31

22. Fernandes AO, Sens YAS, Fonseca Junior PR, Moura RCF, Alves VLS. Impacto do uso do cicloergômetro na função respiratória, cardiovacular, capacidade aeróbica, funcional e qualidade de vida de pacientes com doença renal cronica em hemodiálise. 2019;20(2):302-9. doi: 10.33233/fb.v20i2.2220

23. Hung SC, Kuo KL, Peng $\mathrm{CH}$, Wu CH, Wang YC, Tarng DC. Association of fluid retention with anemia and clinical outcomes among patients with chronic kidney disease. J Am Heart Assoc. 2015;4(1):1-7. doi: 10.1161/JAHA.114.001480

24. Yuen NK, Ananthakrishnan S, Campbell MJ. Hyperparathyroidism of Renal Disease. Perm J. 2016;20(3):15-127. doi: 10.7812/TPP/15-127

25. Sesso RC, Lopes AA, Thomé FS, Lugon JR, Martins CT. Brazilian Chronic Dialysis Survey 2016. J Bras Nefrol. 2017;39(3):261-6. doi: 10.5935/0101-2800.20170049 